\title{
HILBERT SPACE METHODS IN THE THEORY OF LIE ALGEBRAS $\left({ }^{1}\right)$
}

\author{
BY \\ JOHN R. SCHUE
}

Introduction. In the study of the complex finite-dimensional semi-simple Lie algebras a crucial role is played by the fundamental bilinear form $\langle x, y\rangle$ $=\operatorname{Tr}(\operatorname{ad}(x) \operatorname{ad}(y))$. Since the definition is meaningless when the restriction of finite-dimensionality is removed, if any of the highly desirable properties of the form are to be retained in this case they must necessarily be given $a$ priori. By reconsidering the finite-dimensional situation it is possible to formulate suitable conditions in a more convenient form. To see this let $L$ be a complex finite-dimensional semi-simple Lie algebra and let $L_{0}$ be a compact real form for $L$ with $\sigma$ as the associated involution (conjugation). If we let $x^{*}=-\sigma(x)$ and $(x, y)=\left\langle x, y^{*}\right\rangle$ then $L$ becomes a finite-dimensional Hilbert space, the mapping $x$ into $x^{*}$ is a Hilbert space conjugation, and the connecting property $([x, y], z)=\left(y,\left[x^{*}, z\right]\right)$ holds for all $x, y, z$. An $L^{*}$ algebra as defined here is simply a Lie algebra whose vector space is a Hilbert space such that the connecting property above holds. This paper is a study of such algebras with emphasis, of course, on the infinite-dimensional ones. For finite dimensions nothing new is obtained and it is shown here that in this case every semisimple $L^{*}$ algebra arises essentially from a construction like that above (see the remark after 2.5).

There is an associative algebra analogue of this problem in the paper of Ambrose [1] on $H^{*}$ algebras and some of his results are used here. Any $H^{*}$ algebra gives rise to an $L^{*}$ algebra by letting $[x, y]=x y-y x$ and the only known examples of $L^{*}$ algebras are those obtained as Lie subalgebras of $H^{*}$ algebras.

The main result of this paper is a classification of the (separable) simple $L^{*}$ algebras which have Cartan decompositions (see $\$ 2$ ) and it is shown that this class coincides with the simple self-adjoint Lie subalgebras of a (separable) simple $H^{*}$ algebra. The results turn out to be the natural extensions of the finite-dimensional theory.

Associated with each of the Lie algebras considered here there is a gener-

Received by the editors April 9, 1959.

(1) The results in this paper coincide in part with the author's doctoral thesis written at Massachusetts Institute of Technology under the guidance of Professor Kenkichi Iwasawa. The author would like to acknowledge his gratitude to Professor Iwasawa for his considerable patience and interest and his frequent helpful suggestions. 
alized analytic group nucleus. For a discussion of this relationship one may refer to the paper of Birkhoff [2].

\section{Preliminaries.}

Definition. An $L^{*}$ algebra is defined as a Lie algebra $L$ over the complex field such that the vector space of $L$ is a Hilbert space and for each $x \in L$ there is an $x^{*}$ in $L$ with $([x, y], z)=\left(y,\left[x^{*}, z\right]\right)$ for all $y, z$ in $L$.

Examples. Let $A$ be an $H^{*}$ algebra and let $[x, y]=x y-y x$. Any closed Lie subalgebra of $A$ which is closed under the operation of taking adjoints is then an $L^{*}$ algebra. Any complex finite-dimensional semi-simple Lie algebra is an $L^{*}$ algebra. The Hilbert space direct sum of $L^{*}$ algebras defines an $L^{*}$ algebra in the obvious way.

Definitions and Remarks. $L$ will represent an $L^{*}$ algebra. For subsets $M, N$ of $L$ let $M^{*}=\left\{m^{*}: m \in M\right\}, M^{\perp}=\{x:(x, m)=0$ for all $m$ in $M\}$, $[M, N]=$ the closed subspace spanned by $\{[m, n]: m \in M, n \in N\}$. For subspaces $S_{1}$ and $S_{2}$ of $L$ the notation $S_{1}+S_{2}$ will be used only when $S_{1} \perp S_{2}$.

For $x$ in $L$ let $D_{x}$ denote the linear operator $D_{x} y=[x, y]$. Then $D_{x}, D_{x}^{*}$ are everywhere defined (this implies both are bounded) and $D_{x}^{*}=D_{x^{*}}$. By using the principle of uniform boundedness it is not hard to show that the mapping $x$ to $D_{x}$ is continuous from $L$ into the space of bounded operators on $L$ under the uniform norm. Furthermore we may assume that $\left\|D_{x}\right\| \leqq\|x\|$.

$L$ will be called semi-simple if and only if $L=[L, L]$ and this is equivalent to the mapping $x$ to $D_{x}$ being one-one. $L$ will be called simple if and only if there are no nontrivial closed ideals. It is a simple argument to show that a closed subspace $I$ of $L$ is an ideal of $L$ if and only if $I^{\perp}$ is an ideal. Using this one obtains the result that every $L^{*}$ algebra is the direct sum of an abelian ideal (the center) and a semi-simple ideal (the derived algebra, $[L, L]$ ). Hence an $L^{*}$ algebra is necessarily reductive in the sense of [3, Exposé 7 ].

From now on we will assume $L$ is semi-simple. Using the fact that the adjoint representation is then faithful and the properties of adjoints for operators it follows that the mapping $x$ to $x^{*}$ is involutory, conjugate linear, and $[x, y]^{*}=\left[y^{*}, x^{*}\right]$. Then the connecting property implies $(x,[y, z])=\left([y, z]^{*}\right.$, $\left.x^{*}\right)$ for all $x, y, z$. By semi-simplicity, $(x, y)=\left(y^{*}, x^{*}\right)$ for all $x, y$ so that the * mapping is a Hilbert space conjugation. $L$ is then the complexification of the real Lie algebra formed by the skew-adjoint elements. It can be proved from all of this that every closed ideal of $L$ is an $L^{*}$ algebra.

A Cartan subalgebra of a semi-simple $L$ is defined as a maximal selfadjoint abelian subalgebra. An application of Zorn's Lemma shows that every $x \in L$ with $\left[x, x^{*}\right]=0$ is contained in a Cartan subalgebra. A Cartan subalgebra is necessarily closed.

1.1. Let $H$ be a Cartan subalgebra of $L$. Then $H$ is maximal abelian and $H^{\perp}=[H, L]$.

Proof. Suppose $[H, x]=0$. Then $\left[H, x^{*}\right]=\left[H^{*}, x\right]^{*}=[H, x]^{*}=0$. Hence $\left[H, x+x^{*}\right]=\left[H, x-x^{*}\right]=0$. Since $H$ is maximal self-adjoint abelian this im- 
plies $x+x^{*}$ and $x-x^{*}$ are in $H$, hence $x \in H$ and $H$ is maximal abelian. If $h_{1}, h_{2} \in H$ and $x \in L$ then $\left(h_{1},\left[h_{2}, x\right]\right)=\left(\left[h_{2}^{*}, h_{1}\right], x\right)$ implies $\left[h_{2}, x\right] \in H^{\perp}$ and $[H, L] \subset H^{\perp}$. If $x \in[H, L]^{\perp}$ then $\left(x,\left[h^{*}, y\right]\right)=0$ for all $y$ implies $([h, x], y)=0$ so that $[H, x]=0$ and $x \in H$.

In the event that $L$ is finite-dimensional a Cartan subalgebra $H$ as defined here is a Cartan subalgebra in the usual sense. For $H$ is maximal abelian and for each $h \in H,\left[h, h^{*}\right]=0$ implies $D_{h}$ is normal, hence diagonalizable. These two properties characterize the Cartan subalgebras of $L$ (see [3, Exposé 9]). Conversely, if $L$ is semi-simple and finite-dimensional, a Cartan subalgebra $H$ of $L$ in the sense of [3] is one in our sense for a suitable * mapping and inner product, for by Exposé 11 of [3] there is a compact real form $L_{0}$ of $L$ with associated involution $\sigma$ such that $\sigma(H)=H$. Applying the construction used in the introduction gives the result.

1.2. Theorem 1. Let $L$ be a semi-simple $L^{*}$ algebra. Then there exist simple closed $L^{*}$ ideals $L_{j}$, indexed by some set $J$, such that $L=\sum_{j \in J} L_{j}$, the sum being the usual Hilbert space direct sum. Every closed ideal of $L$ is obtained by summing the $L_{j}$ over some subset of $J$.

Outline of Proof. Let $H$ be a Cartan subalgebra of $L$ and $B$ the $C^{*}$ algebra generated by $\left\{D_{h}: h \in H\right\} . B$ is then topologically and algebraically isomorphic with the algebra of all continuous complex-valued functions vanishing at infinity on the locally compact space $\Delta$ of all homomorphisms of $B$ onto the complex numbers. Each $\alpha \in \Delta$ defines a bounded linear functional on $H$ and hence there is a unique $h_{\alpha} \neq 0$ in $H$ such that $\alpha\left(D_{h}\right)=\left(h, h_{\alpha}\right)$ for all $h$. Then $\left\|h_{\alpha}\right\| \leqq 1$ and $\alpha\left(D_{h}^{*}\right)=\left[\alpha\left(D_{h}\right)\right]^{-}$implies $h_{\alpha}^{*}=h_{\alpha}$. For $\alpha, \beta \in \Delta$ let $(\alpha, \beta)=\left(h_{\alpha}, h_{\beta}\right)$ and definite $\alpha \perp \beta$ if and only if $(\alpha, \beta)=0$. A subset $M$ of $\Delta$ will be called indecomposable if $M$ cannot be written as the union of nonempty orthogonal subsets. Then each $\alpha \in \Delta$ is contained in a unique maximal indecomposable subset $M_{\alpha}$. Then either $M_{\alpha}=M_{\beta}$ or $M_{\alpha} \perp M_{\beta}$. Let $\left\{M_{j}: j \in J\right\}$ be the set of the distinct $M_{\alpha}$ 's. For each $j$ let $H_{j}$ be the span of the $h_{\alpha}$ where $\alpha$ runs over $M_{j}$ and let $L_{j}=H_{j}+\left[H_{j}, L\right]$. By a proof like that used in the finite-dimensional case each $L_{j}$ is a simple closed ideal of $L$ and $L_{j} \perp L_{k}$ for $j \neq k$. If $K=\sum L_{j}$ then $K$ is a closed ideal containing $H$ (the $h_{\alpha}$ 's span $H$ ) and hence $\left[K^{\perp}, H\right]=0$ implies $K^{\perp}=0$ so that $L=\sum L_{j}$. The last statement is a consequence of the way the decomposition is obtained.

\section{Roots and Cartan decompositions.}

Definition. For this section $L$ is a semi-simple $L^{*}$ algebra with $H$ as a Cartan subalgebra. For a linear mapping $\alpha$ of $H$ into the complex numbers let $V_{\alpha}=\{v:[h, v]=\alpha(h) v$ for all $h \in H\}$. Then $V_{\alpha}$ is a closed subspace of $L$ and $\alpha$ will be called a root (relative to $H$ ) if and only if $V_{\alpha} \neq 0$. The zero function is a root and $V_{0}=H$. If $\alpha$ is a root then necessarily it corresponds to a homomorphism of the operator algebra generated by $\left\{D_{h}: h \in H\right\}$. Hence $\alpha$ is bounded and $\alpha\left(h^{*}\right)=[\alpha(h)]^{-}$. As in the proof of Theorem 1 there is a unique 
$h_{\alpha}$ in $H$ with $\left\|h_{\alpha}\right\| \leqq 1, h_{\alpha}^{*}=h_{\alpha}$, and $\alpha(h)=\left(h, h_{\alpha}\right)$ for all $h$. From this it follows that if $\alpha$ is a root $-\alpha$ is also one and $V_{\alpha}^{*}=V_{-\alpha}$. If $\alpha, \beta$ are distinct then $V_{\alpha} \perp V_{\beta}$. By the Jacobi identity $\left[V_{\alpha}, V_{\beta}\right] \subset V_{\alpha+\beta}$.

Let $K=\sum V_{\alpha}$, the sum being taken over the distinct roots relative to $H$. Then $K$ is a closed $L^{*}$ subalgebra of $L$ with $H \subset K \subset L$. We will say that $L$ has a Cartan decomposition (relative to $H$ ) if and only if $K=L$, i.e. if and only if the set $\left\{D_{h}: h \in H\right\}$ is simultaneously diagonalizable. It is an open question as to whether or not every $L$ has such a decomposition; however, I hope to have more complete results to be given in a later paper. Theorem 2 below settles the question if $L$ is embedded in an $H^{*}$ algebra and the later classification theory shows this is necessary as well as sufficient, at least when every simple ideal component of $L$ is separable.

2.1. Let $L$ be a simple $L^{*}$ algebra and suppose $\phi$ is a continuous linear mapping of $L$ into a Hilbert space $K$ with $(\phi([x, y]), \phi(z))=\left(\phi(y), \phi\left(\left[x^{*}, z\right]\right)\right)$ for all $x, y, z$ in $L$. Then there is an $\epsilon \geqq 0$ such that $(\phi(x), \phi(y))=\epsilon(x, y)$ for $x$ and $y$ in $L$.

Proof. Since $\phi$ is bounded there is a bounded operator $B$ on $L$ such that $(\phi(x), \phi(y))=(B x, y)$. Then $B \geqq 0$ implies $B$ is self-adjoint. The assumption on $\phi$ implies $B$ commutes with every $D_{x}$; by the spectral theorem every projection in the spectral resolution of $B$ commutes with every $D_{x}$. The range of such a projection is then a closed ideal of $L$, hence is either 0 or all of $L$ so that $B=\epsilon 1$ for some $\epsilon \geqq 0$.

2.2. Theorem 2. Suppose $L$ is a semi-simple $L^{*}$ subalgebra of an $H^{*}$ algebra $A$ and $H$ is a Cartan subalgebra of $L$. Then $L$ has a Cartan decomposition relative to $H$.

Proof. Let $L=\sum L_{j}$ where each $L_{j}$ is a simple closed ideal. If $H_{j}=H \cap L_{j}$ it is easily seen that $H_{j}$ is a Cartan subalgebra of $L_{j}$. Hence it will be sufficient to prove the theorem when $L$ is simple.

If $I$ is a simple (associative) ideal of $A$ the restriction to $L$ of the projection $P$ of $A$ onto $I$ satisfies the hypotheses of 2.1 and hence there is an $\epsilon \geqq 0$ such that $(P x, P y)=\epsilon(x, y)$ for all $x, y$ in $L$. Since $A$ is a direct sum of such simple ideals there must be some $I$ such that the corresponding $\epsilon$ is positive. Thus $L$ is topologically and algebraically isomorphic with a Lie subalgebra of $I$ so that we may assume $A$ itself is simple. Then by [1], $A$ is the set of all Hilbert-Schmidt operators on some Hilbert space $\mathcal{H}$.

The set $H$ is then a collection of commutative completely continuous normal operators on $\mathcal{H}$ and hence can be simultaneously diagonalized. Using a basis of $\mathscr{H}$ composed of common eigenvectors for $H$ and regarding $A$ as the algebra of square-convergent matrices relative to this basis, $H$ becomes a subset of the diagonal matrices. For $h \in H$ and $y \in A$ let $T_{h} y=h y-y h$. Then, as in the finite-dimensional case, the operators $T_{h}$ can be simultaneously 
diagonalized. Since $L$ is an invariant subspace under the set of all $T_{h}$ and the restriction of $T_{h}$ to $L$ is $D_{h}$ then $L$ has a Cartan decomposition relative to $H$.

For the remainder of this section we will assume only that $L$ is semisimple and $H$ is a Cartan subalgebra.

2.3. If $\alpha$ is a nonzero root $V_{\alpha}$ is one-dimensional.

Proof. Choose $v_{1} \in V_{\alpha}$ with $\left\|v_{1}\right\|=1$. Let $v_{2} \in V_{\alpha}$ with $\left(v_{1}, v_{2}\right)=0$. It is sufficient to show that this implies $v_{2}=0$. For any $v \in V_{\alpha}$ we have $\left[v_{1}, v^{*}\right] \in H$. For any $h \in H,\left(h,\left[v_{1}, v^{*}\right]\right)=\left([h, v], v_{1}\right)=\left(h, h_{\alpha}\right)\left(v, v_{1}\right)$ implies $\left[v_{1}, v^{*}\right]=\left(v_{1}, v\right) h_{\alpha}$ so that $\left[v_{1}, v_{2}^{*}\right]=0$. The same argument can be used to show that $\left[v_{2}, v_{2}^{*}\right]$ $=\left\|v_{2}\right\|^{2} h_{\alpha}$. Then, by the Jacobi identity and the connecting property, $0=\left(\left[v_{1}^{*}, v_{2}\right], \quad\left[v_{1}^{*}, v_{2}\right]\right)=\left(\left[v_{2}, v_{2}^{*}\right], \quad\left[v_{1}, v_{1}^{*}\right]\right)+\left(\left[v_{1}, v_{2}\right], \quad\left[v_{1}, v_{2}\right]\right)=\left\|v_{2}\right\|\left\|^{2}\right\| h_{\alpha} \|^{2}$ $+\left\|\left[v_{1}, v_{2}\right]\right\|^{2}$ so that $\left\|v_{2}\right\|=0$ and $v_{2}=0$.

Definition. Let $R$ be the set of nonzero roots relative to $H$. By Zorn's lemma it is possible to decompose $R$ as $R=R_{1} \cup R_{2}$ where $R_{1}, R_{2}$ are disjoint and $\alpha \in R_{1}$ if and only if $-\alpha \in R_{2}$. For each $\alpha \in R_{1}$ choose $e_{\alpha} \in V_{\alpha}$ such that $\left\|e_{\alpha}\right\|=1$. Then $e_{\alpha}^{*} \in V_{-\alpha}$ and $\left\|e_{\alpha}^{*}\right\|=1$. For $\alpha \in R_{2}$ let $e_{\alpha}=e_{-\alpha}^{*}$. Thus $e_{\alpha}^{*}=e_{-\alpha}$ for all $\alpha$ in $R$ and the set $\left\{e_{\alpha}\right\}$ is an orthonormal set. By the proof of $2.2,\left[e_{\alpha}, e_{\alpha}^{*}\right]$ $=h_{\alpha}$.

Suppose $\alpha, \beta \in R$ and $\beta \neq-\alpha$. If $\alpha+\beta$ is a root let $c_{\alpha, \beta}$ be defined by the equation $\left[e_{\alpha}, e_{\beta}\right]=c_{\alpha, \beta} e_{\alpha+\beta}$, otherwise let $c_{\alpha, \beta}=0$ and $e_{\alpha+\beta}=0$.

If $\beta$ is any root and $\alpha$ a nonzero root the sequence $\{\beta-k \alpha: k=0, \pm 1, \ldots\}$ contains only finitely many roots for if $\beta-k \alpha$ is a root then $1 \geqq\left\|h_{\beta-k \alpha}\right\|$ $=\left\|h_{\beta}-k h_{\alpha}\right\| \geqq|k|\left\|h_{\alpha}\right\|-\left\|h_{\beta}\right\|$. Thus it is possible to define the integers $k_{1}(\alpha, \beta)$ and $k_{2}(\alpha, \beta)$ by the conditions $\beta+k \alpha$ is a root for $-k_{1} \leqq k \leqq k_{2}$ while $\beta-\left(k_{1}+1\right) \alpha$ and $\beta+\left(k_{2}+1\right)$ are not roots. Then, by the same proof as used in [3], $\left(h_{\alpha}, h_{\beta}\right)=(1 / 2)\left[k_{1}(\alpha, \beta)-k_{2}(\alpha, \beta)\right]\left\|h_{\alpha}\right\|^{2}$ for any roots $\alpha, \beta$ with $\alpha \neq 0$.

2.4. Suppose $\alpha_{1}, \cdots, \alpha_{k} \in R$. Let $M$ be the set of all roots which are linear combinations with integral coefficients of $\alpha_{1}, \cdots, \alpha_{k}$. Let $V$ be the span of the $e_{\alpha}$ 's where $\alpha \in M$ and let $H_{1}$ be the span of $h_{\alpha_{1}}, \cdots, h_{\alpha_{k}}$. Then $L_{1}=H_{1}+V$ is a finite-dimensional semi-simple $L^{*}$ algebra with $H_{1}$ as a Cartan subalgebra and $M$ is the complete set of roots relative to $H_{1}$.

Proof. The proof is straightforward except, perhaps, for the statement that the dimension of $L_{1}$ is finite. Since $\operatorname{dim} H_{1} \leqq k<\infty, \operatorname{dim} L_{1}$ is infinite if and only if $\left\{e_{\alpha}: \alpha \in M\right\}$ is infinite and this can occur only if $\left\{h_{\alpha}: \alpha \in M\right\}$ is infinite. In this event the latter set is an infinite bounded set in the unitary space $H_{1}$ and must then contain an infinite convergent sequence $h_{\beta_{n}}$. Letting $h_{i}$ $=2\left\|h_{\alpha_{i}}\right\|^{-2} h_{\alpha_{i}}$ for $i=1, \cdots, k,\left(h_{\beta_{n}}, h_{i}\right)$ is an integer for all $n$ and $i$ and $H_{1}$ is spanned by $h_{1}, \cdots, h_{k}$. From this it is clear that no such sequence exists and consequently $L_{1}$ is finite-dimensional.

2.5. Suppose $L$ is finite-dimensional and simple. Let $\langle x, y\rangle=\operatorname{Tr}\left(D_{x} D_{y}\right)$ for all $x, y$. Then there is an $\epsilon>0$ such that $\langle x, y\rangle=\left(x, y^{*}\right)$.

Proof. Define the operator $B$ on $L$ by the equation $(B x, y)=\left\langle x, y^{*}\right\rangle$ for all 
$x, y$. Then $(B x, x)=\operatorname{Tr}\left(D_{x} D_{x}^{*}\right)$ implies $B$ is positive definite. The condition $\langle[x, y], z\rangle=\langle x,[y, z]\rangle$ implies $B$ commutes with every $D_{x}$ and, by the argument used in 2.1, $B$ must be a positive multiple of the identity.

REMARK. The result of 2.5 justifies the remarks of the introduction for finite-dimensional $L^{*}$ algebras. If $L$ is simple and $\epsilon$ is as in 2.5 let $L_{0}$ be the set of skew-adjoint elements of $L$ and $\sigma(x)=-x^{*}$ for all $x$. Then $\sigma$ is an involution and 2.5 shows that $L_{0}$ is a compact real form for $L$. The extension to semi-simple algebras is immediate. An immediate consequence of this relationship is the result 2.6 below which will be needed in the later classification theory.

2.6. Let $L$ be finite-dimensional and simple with $H$ as a Cartan subalgebra.

(i) If $\alpha, \beta, \gamma \in R$ and $\alpha+\beta+\gamma=0$ then $c_{\alpha, \beta}=c_{\beta, \gamma}=c_{\gamma, \alpha}$.

(ii) If $\alpha, \beta, \gamma, \delta \in R, \alpha+\beta+\gamma+\delta=0$, and the sum of no pair is zero, then $c_{\alpha, \beta} c_{\gamma, \delta}+c_{\beta, \gamma} c_{\alpha, \delta}+c_{\gamma, \alpha} c_{\beta, \delta}=0$.

(iii) If $\alpha, \beta \in R$ and $\beta \neq-\alpha$ then

$$
c_{\alpha, \beta} c_{-\alpha,-\beta}=-(1 / 2) k_{2}(\alpha, \beta)\left(1+k_{1}(\alpha, \beta)\right)\left\|h_{\alpha}\right\|^{2} .
$$

Proof. See [3, Exposé 11, Lemmas 1, 2, 3].

3 . The classification theory. For this section $L$ will be a simple infinitedimensional $L^{*}$ algebra and $H$ a Cartan subalgebra such that $L$ has a Cartan decomposition relative to $H$. We further require that the space of $L$ be separable.

Definition. For a finite subset $F=\left\{\alpha_{1}, \cdots, \alpha_{k}\right\}$ of $R$ let $L(F)$ denote the finite-dimensional semi-simple algebra defined in 2.4. Then $F_{1} \subset F_{2}$ implies $L\left(F_{1}\right) \subset L\left(F_{2}\right)$. A subset $G$ of $R$ will be called a root system if and only if $\alpha \in G$ implies $-\alpha \in G$ and $\alpha, \beta \in G, \alpha+\beta \in R$ implies $\alpha+\beta \in G$. Then $L(F)$ is the subalgebra generated by the $e_{\alpha}$ where $\alpha$ ranges over the root system generated by $F$. Using the notion of indecomposability as in the proof of Theorem 1 , if $F$ is an indecomposable finite subset of $R$ then the root system generated by $F$ is indecomposable and $L(F)$ is simple. Furthermore it is clear that $R$ is indecomposable since $L$ is simple. A subset $\alpha_{0}, \cdots, \alpha_{n}$ of $R$ will be called a chain from $\alpha_{0}$ to $\alpha_{\alpha}$ if $\left(h_{\alpha_{i-1}}, h_{\alpha_{i}}\right) \neq 0$ for $i=1, \cdots, n$. Since $R$ is indecomposable any $\alpha, \beta \in R$ must be connected by a finite chain. Any chain is indecomposable.

3.1. For any finite subset $F$ of $R$ there exists a finite indecomposable root system containing $F$.

Proof. Let $F=\left\{\alpha_{1}, \cdots, \alpha_{n}\right\}$. For each $i, 1 \leqq i \leqq n-1$, let $F_{i}$ be a chain from $\alpha_{i}$ to $\alpha_{i+1}$. Let $F_{1}=\bigcup F_{i}$. Then $F_{1}$ is indecomposable and finite. If $F_{2}$ is the root system generated by $F_{1}, F_{2}$ is indecomposable and 2.4 implies $F_{2}$ is finite.

Definition. Since $L$ is separable the orthonormal set $\left\{e_{\alpha}: \alpha \in R\right\}$ is count- 
able and hence $R$ is countably infinite. Let $R=\left\{\alpha_{1}, \alpha_{2}, \cdots\right\}$ and let $F_{n}$ $=\left\{\alpha_{1}, \cdots, \alpha_{n}\right\}$.

3.2. There is a sequence $G_{n}$ of finite subsets of $R$ such that the following are true:

(i) $F_{n} \subset G_{n} \subset G_{n+1}$.

(ii) $G_{n}$ is an indecomposable root system.

(iii) $R=\bigcup G_{n}$.

(iv) The simple subalgebras $L\left(G_{n}\right)$ form a strictly increasing sequence with $L=$ closure of $\mathrm{U} L\left(G_{n}\right)$. All of the $L\left(G_{n}\right)$ are of the same Cartan type A, $\mathrm{B}, \mathrm{C}$, or D.

Proof. The sequence $\left\{G_{n}\right\}$ can be defined inductively. Let $G_{1}$ be a finite indecomposable root system containing $F_{1}$. Having chosen $G_{1}, \cdots, G_{n-1}$ satisfying (i) and (ii) let $F=G_{n-1} \cup F_{n}$ and choose $G_{n}$ to be a finite indecomposable root system containing $F$. The $G_{n}$ obtained in this way will then satisfy (i) and (ii). Since $R=\bigcup F_{n}$, (iii) will hold and $G_{n} \subset G_{n+1}$ implies $L\left(G_{n}\right)$ $C L\left(G_{n+1}\right)$. An $h \in H$ such that $\left(h, h_{\alpha}\right)=0$ for all $\alpha$ in $R$ would then have $D_{h}=0$, hence $h=0$ and $H$ is spanned by the set of $h_{\alpha}$. Since the set of $e_{\alpha}$ spans $H^{\perp}$ then $L=$ closure of $U L\left(G_{n}\right)$. Now $\operatorname{dim} L$ is infinite and each $L\left(G_{n}\right)$ is finite-dimensional so that there are infinitely many distinct $L\left(G_{n}\right)$. Then any infinite subsequence of the $G_{n}$ will also satisfy (i)-(iii) and the first part of (iv). By passing to subsequences if necessary it is possible to eliminate any duplications and furthermore obtain a sequence whose elements are all of the same type. Since their dimensions are unbounded there can be no exceptional algebras.

Definition. Let $K_{n}$ be the real linear subspace of the conjugate space of $H$ spanned by $\left\{\alpha: \alpha \in G_{n}\right\}$. Let $p_{1}=\operatorname{dim} K_{1}$ and $p_{n}=\operatorname{dim}\left(K_{n} / K_{n-1}\right)$ for $n=2,3, \cdots$. Then each $p_{i}$ is a positive integer and the rank of the simple algebra $L\left(G_{n}\right)$ is $p_{1}+\cdots+p_{n}$.

Since $G_{1}$ is a root system for $L\left(G_{1}\right)$ there exist $\alpha_{1,1}, \cdots, \alpha_{1, p_{1}}$ in $G_{1}$ which form a linear basis of $K_{1}$. Since $G_{2}$ is a root system for $L\left(G_{2}\right)$ there exist $\alpha_{2,1}, \cdots, \alpha_{2, p_{2}}$ in $G_{2}$ such that $\alpha_{1,1}, \cdots, \alpha_{1, p_{1}}, \alpha_{2,1}, \cdots, \alpha_{2, p_{2}}$ form a linear basis for $K_{2}$. Necessarily $\alpha_{2, i} \notin G_{1}$. Continuing this process we can find, for each $n \geqq 2, \alpha_{n, 1}, \cdots, \alpha_{n, p_{n}} \in G_{n}-G_{n-1}$ such that the set

$$
\left\{\alpha_{i, j}: i=1, \cdots, n ; j=1, \cdots, p_{i}\right\}
$$

is a linear basis for $K_{n}$. Order this basis as follows:

$$
\alpha_{n, p_{n}}, \cdots, \alpha_{n, 1}, \alpha_{n-1, p_{n-1}}, \cdots, \alpha_{n-1,1}, \cdots, \alpha_{2,1}, \alpha_{1, p_{1}}, \cdots, \alpha_{1,1} .
$$

Suppose $\tau \in K_{n}, \tau \neq 0$. Then $\tau$ is a linear combination with real coefficients of the elements of this ordered basis. Define $\tau>0$ or $\tau<0$ according as the first nonzero coefficient is positive or negative. For $\tau_{1} \neq \tau_{2}$ let $\tau_{1}>\tau_{2}$ if and only if $\tau_{1}-\tau_{2}>0$. This then gives a total ordering of $K_{n}$ and induces an order- 
ing of $G_{n}$. By the choice of basis for each $K_{n}$, for integers $n, m$ and $\alpha, \beta \in G_{n}$ $\cap G_{m}, \alpha>\beta$ in the ordering of $G_{n}$ if and only if $\alpha>\beta$ in the ordering of $G_{m}$.

Now suppose $\alpha, \beta$ are any roots. Choose $n$ such that $\alpha, \beta \in G_{n}$ and define $\alpha>\beta$ if and only if they are so related in the ordering of $G_{n}$. This gives a welldefined total ordering on the set of all roots and has the following properties:

(i) $\alpha>0$ implies $-\alpha<0$.

(ii) $\alpha>0, \beta>0$ implies $\alpha+\beta>0$.

(iii) If $\alpha>0$ and $\alpha \notin G_{n}$ then $\alpha>\beta$ for every $\beta \in G_{n}$.

(iv) The ordering induced on $G_{n}$ is a lexicographical ordering with respect to a basis of roots.

Let $R^{+}$be the set of positive roots. Then, since $G_{n}$ is finite, property (iii) implies that $R^{+}$is well-ordered. An $\alpha \in R^{+}$will be called simple if $\alpha$ cannot be written as the sum of two positive roots. Let $S$ denote the set of all simple roots.

3.3. (1) $S \cap G_{n}$ is a complete set of simple roots (in the sense of [3]) for $L\left(G_{n}\right)$.

(2) For $\alpha, \beta$ in $S, \alpha-\beta$ is a root only if $\alpha=\beta$. Thus $k_{1}(\alpha, \beta)=k_{1}(\beta, \alpha)=0$.

(3) $S$ is linearly independent over the reals and every $\alpha$ in $R^{+}$is a linear combination of elements of $S$ with non-negative integral coefficients which are almost all zero.

(4) If $\tau=\sum n_{i} \alpha_{i}$ where $\alpha_{i} \in S$ and almost all $n_{i}$ are zero there is an algorithm to determine whether or not $\tau$ is a root. To apply the algorithm it is sufficient to know $\left(h_{\alpha}, h_{\beta}\right)$ for all $\alpha, \beta \in S$.

Proof. (1) If $\alpha, \beta, \gamma \in R^{+}$and $\alpha=\beta+\gamma$ then $\alpha>\beta>0$ and $\alpha>\gamma>0$. If $\alpha \in G_{n}$ then (iii) of the definition above implies $\beta, \gamma \in G_{n}$. Hence an $\alpha \in G_{n}$ is simple in $G_{n}$ if and only if $\alpha$ is simple in $R$.

(2) and (3) can be deduced from the corresponding properties for the finite-dimensional case proved in [3, Exposé 10].

(4) Choose $n$ such that $\tau \in K_{n}$. The statement then follows from the result proved in [3, Expose 16], applied to the algebra $L\left(G_{n}\right)$, using the fact that the fundamental bilinear form is determined up to a constant multiple.

Definition. Define the graph of $S$ to be the set $G$ of all $\left(h_{\alpha}, h_{\beta}\right)$ where $\alpha, \beta$ vary over $S$. Then knowing the graph is equivalent to determining $\left\|h_{\alpha}\right\|$ and $k_{2}(\alpha, \beta)$ for $\alpha, \beta$ in $S$. If $L, L^{\prime}$ are two algebras of the type considered in this section with $H$ and $H^{\prime}$ as Cartan subalgebras and $G, G^{\prime}$ as the corresponding graphs we will say that $G$ is isomorphic to $G^{\prime}$ if and only if there is a mapping $\alpha$ to $\alpha^{\prime}$ of $S$ onto $S^{\prime}$ with $\left(h_{\alpha}, h_{\beta}\right)=\left(h_{\alpha^{\prime}}^{\prime}, h_{\beta^{\prime}}^{\prime}\right)$ for all $\alpha, \beta$ in $S$.

3.4. Let $L, L^{\prime}$ be as above and suppose $G$ is isomorphic to $G^{\prime}$. Then there is an algebraic isomorphism $\phi$ of $L$ onto $L^{\prime}$ such that:

(1) $\phi\left(h_{\alpha}\right)=h_{\alpha^{\prime}}^{\prime}$ for all $\alpha \in R$.

(2) $\phi(x)^{*}=\phi\left(x^{*}\right)$ for all $x \in L$.

(3) $(\phi(x), \phi(y))=(x, y)$ for all $x, y$ in $L$.

Proof. By using the algorithm of 3.3, (4) it is possible to extend the map of $S$ onto $S^{\prime}$ to a mapping $\alpha$ to $\alpha^{\prime}$ of $R$ onto $R^{\prime}$ which preserves inner products 
for the $h_{\alpha}$. This mapping then necessarily preserves all of the algebraic structure of $R$. For a complex linear combination $h=\sum c_{i} h_{\alpha_{i}}$, where $\alpha_{i} \in R$, let $\phi(h)=\sum c_{i} h_{\alpha_{\mathbf{i}^{\prime}}}^{\prime}$. Then $\phi$ is well-defined and preserves inner products so that it extends uniquely to an isometry of $H$ onto $H^{\prime}$ and satisfies (1). Since $\phi\left(h_{\alpha}^{*}\right)$ $=\phi\left(h_{\alpha}\right)^{*}$ for all $h_{\alpha}, \phi$ will satisfy (2) for any $x \in H$.

Let $\left\{f_{\alpha^{\prime}}: \alpha^{\prime} \in R^{\prime}\right\}$ be a fixed set of elements in $L^{\prime}$ with $f_{\alpha^{\prime}} \in V_{\alpha^{\prime}},\left\|f_{\alpha^{\prime}}\right\|=1$, and $f_{\alpha^{\prime}}^{*}=f_{-\alpha^{\prime}}$. Let $c_{\alpha^{\prime}, \beta^{\prime}}$ be the structure constants for $L^{\prime}$ defined by the set of $f_{\alpha^{\prime}}$. To extend $\phi$ to all of $L$ with the required properties it is then sufficient to find a set $\left\{e_{\alpha}: \alpha \in R\right\}$ in $L$ with $e_{\alpha} \in V_{\alpha},\left\|e_{\alpha}\right\|=1, e_{\alpha}^{*}=e_{-\alpha}$, and such that the structure constants $c_{\alpha, \beta}$ for $L$ defined by this set satisfy $c_{\alpha, \beta}=c_{\alpha^{\prime}, \beta^{\prime}}$.

Thus the problem is reduced to finding the set of $e_{\alpha}$. A corresponding result appears in [3, Exposé 11, Théorème 1]. An examination of the proof there shows the essential features are a well-ordering of $R^{+}$compatible with the algebraic structure and the relations on the structure constants which were proved here in 2.6. (These hold for $L$ since there is always an $n$ such that $\alpha, \beta, \gamma, \delta$ all lie in $G_{n}$.) Using these, the proof in [3] can be repeated here word for word.

3.5. Because of 3.4 it only remains to determine the possible graphs for $L$ and give examples of each type in order to complete the classification.

First, suppose all of the $L\left(G_{n}\right)$ in 3.2 are of type A. Then the root diagram for the simple system $S \cap G_{1}$ has the form:

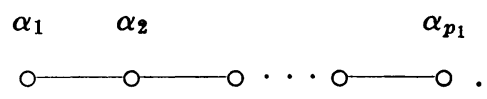

This means, of course, that $k_{2}\left(\alpha_{i}, \alpha_{i-1}\right)=k_{2}\left(\alpha_{i}, \alpha_{i+1}\right)=1$ and otherwise $k_{2}\left(\alpha_{i}, \alpha_{j}\right)=0$. Furthermore, by the remark after $2.5,\left\|h_{\alpha_{i}}\right\|=\left\|h_{\alpha_{j}}\right\|$ for $1 \leqq i$, $j \leqq p_{1}$. Now let $S \cap G_{2}$ be written as $\alpha_{1}, \cdots, \alpha_{p_{1}}, \beta_{1}, \cdots, \beta_{p_{2}}$. After any necessary reordering the diagram for $S \cap G_{2}$ will have the form:

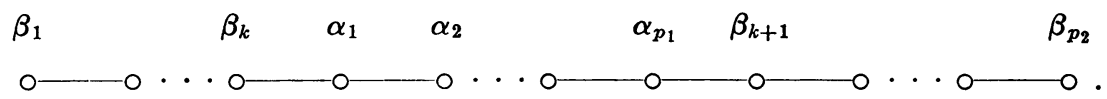

It is possible that all the $\beta_{j}$ may be at one end of the chain. Again it follows that $\left\|h_{\alpha_{i}}\right\|=\left\|h_{\beta_{j}}\right\|$ for $\alpha_{i}, \beta_{j}$. Continuing this process and introducing the necessary new notation for the $\alpha$ 's in $S$ we will obtain one of the following two possibilities:

Type A.

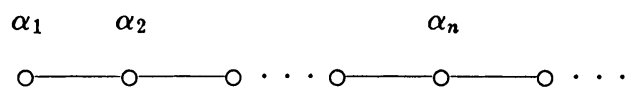

or

Type $A^{\prime}$.

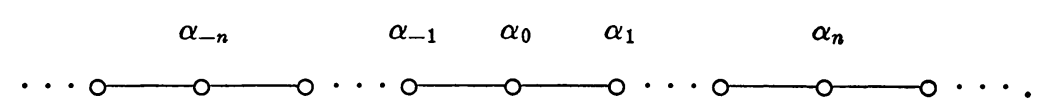


In either case $\left\|h_{\alpha_{i}}\right\|=\left\|h_{\alpha_{j}}\right\|$ for all $i, j, k_{2}\left(\alpha_{i}, \alpha_{j}\right)=0$ for $j \neq i-1, i+1$ while $k_{2}\left(\alpha_{i}, \alpha_{i-1}\right)=k_{2}\left(\alpha_{i}, \alpha_{i+1}\right)=1$. Thus the graph is completely determined up to a constant multiple.

Entirely similar arguments for the other possibilities give the following types:

Type B.

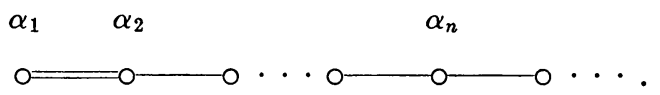

Here $2^{1 / 2}\left\|h_{\alpha_{1}}\right\|=\left\|h_{\alpha_{i}}\right\|$ for $i=2,3, \cdots$ and $k_{2}\left(\alpha_{1}, \alpha_{2}\right)=1, k_{2}\left(\alpha_{2}, \alpha_{1}\right)=1$ while otherwise $k_{2}\left(\alpha_{i}, \alpha_{j}\right)$ is as above.

Type C.

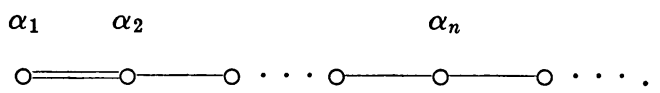

Here $\left\|h_{\alpha_{1}}\right\|=2^{1 / 2}\left\|h_{\alpha_{i}}\right\|$ for $i=2,3, \cdots$ and $k_{2}\left(\alpha_{1}, \alpha_{2}\right)=2, k_{2}\left(\alpha_{2}, \alpha_{1}\right)=1$ while otherwise $k_{2}\left(\alpha_{i}, \alpha_{j}\right)$ is as above.

Type D.

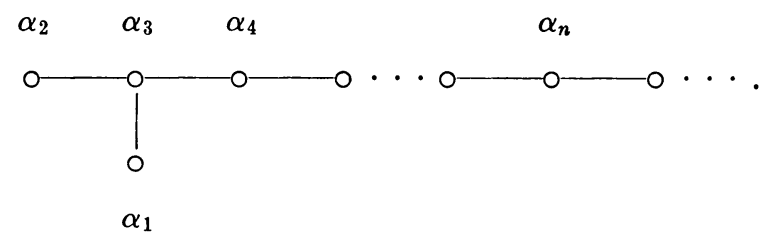

Here $\left\|h_{\alpha_{i}}\right\|=\left\|h_{\alpha_{j}}\right\|$ for all $i$ and $j$ and $k_{2}\left(\alpha_{1}, \alpha_{2}\right)=k\left(\alpha_{2}, \alpha_{1}\right)=0, k_{2}\left(\alpha_{3}, \alpha_{1}\right)=1$ while otherwise $k_{2}\left(\alpha_{i}, \alpha_{j}\right)$ is as above.

3.6. In this paragraph it will be shown that each of the five types $A, A^{\prime}$, B, C, D occurs as the graph of an $L^{*}$ algebra. However, these algebras are not all distinct and give rise to only three nonisomorphic types. More explicitly, $\mathrm{A}$ and $\mathrm{A}^{\prime}$ are isomorphic and so are $\mathrm{B}$ and $\mathrm{D}$.

All of these examples are Lie subalgebras of the associative $H^{*}$ algebra $K$ of Hilbert-Schmidt operators on a separable Hilbert space $\mathfrak{H C}$. For descriptive purposes it is convenient to choose an orthonormal basis of $\mathcal{H C}$ and regard $K$ as a matrix algebra relative to this basis. In each case a Cartan subalgebra $H$ is obtained by taking the intersection of the algebra in question with the set of diagonal matrices. Having done this we will let $\lambda_{i}$ denote the linear functional on $H$ which assigns the $i$ th diagonal entry to every element of $H$. Determination of a set of simple roots and the associated graph is analogous to the finite-dimensional case and the computations will be omitted here. After choosing the proper norm on $L$ an application of 3.4 and 3.5 will show that $L$ is isomorphic in all respects to one of the algebras described here.

In the following discussion a conjugate linear transformation $J$ of $\mathfrak{H C}$ onto 
FC such that $(J x, J y)=(y, x)$ will be called a conjugation if $J^{2}=1$ and an anti-conjugation if $J^{2}=-1$.

Type A. Let $\left\{\phi_{n}: n=1,2, \cdots\right\}$ be a basis of $\mathfrak{F C}$ and let $A$ be the Lie algebra of all Hilbert-Schmidt matrices relative to this basis. $A$ is simple since the center of $K$ is trivial. A simple system of roots is given by

$$
\left\{\lambda_{i}-\lambda_{i+1}: i=1,2, \cdots\right\} \text {. }
$$

Type $A^{\prime}$. Let $\left\{\phi_{n}: n=0, \pm 1, \pm 2, \cdots\right\}$ be a basis of $\mathcal{H C}$ and let $\mathrm{A}^{\prime}$ be the Lie algebra of all Hilbert-Schmidt matrices relative to this basis. A simple system of roots is given by $\left\{\lambda_{i}-\lambda_{i+1}: i=0, \pm 1, \pm 2, \cdots\right\}$.

The algebras $A$ and $A^{\prime}$ are isomorphic since there is a unitary operator $U$ on $\mathfrak{F}$ such that $X \in A$ if and only if $U X U^{-1}$ is in $A^{\prime}$.

TyPe B. Let $\left\{\phi_{n}: n=0, \pm 1, \pm 2, \cdots\right\}$ be a basis of $\mathcal{H C}$ and let $J_{1}$ be the conjugation of $\mathcal{F C}$ such that $J_{1} \phi_{n}=\phi_{-n}$. Let $B$ be the set of Hilbert-Schmidt operators $T$ such that $T^{*} J_{1}=-J_{1} T$. If $\langle x, y\rangle$ is defined by $\langle x, y\rangle=\left(x, J_{1} y\right)$ for $x, y \in \mathfrak{F}$ then $\langle$,$\rangle is a symmetric bilinear form and B$ is the set of $T$ in $K$ which are skew-adjoint with respect to this form. A simple system of roots is given by $\left\{\lambda_{1}, \lambda_{i}-\lambda_{i+1}: i=1,2, \cdots\right\}$.

Type D. Let $\left\{\phi_{n}: n= \pm 1, \pm 2, \cdots\right\}$ be a basis of $\mathfrak{H C}$ and let $J_{2}$ be the conjugation on $\mathcal{H C}$ such that $J_{2} \phi_{n}=\phi_{-n}$. Let $D$ be the set of $T$ in $K$ such that $T^{*} J_{2}=-J_{2} T$. A simple system of roots is given by

$$
\left\{\lambda_{1}+\lambda_{2}, \lambda_{i}-\lambda_{i+1}: i=1,2, \cdots\right\} .
$$

Since $J_{1}$ and $J_{2}$ are two conjugations of $\mathfrak{H}$ there is a unitary $U$ on $\mathscr{C}$ such that $U J_{1}=J_{2} U$. Then for any $T \in K, T \in B$ if and only if $U T U^{-1}$ is in $D$. Hence $B$ is isomorphic to $D$.

Type C. Let $\left\{\phi_{n}: n= \pm 1, \pm 2, \cdots\right\}$ be a basis of $\mathcal{H}$. Let $J$ be the anticonjugation on $\mathcal{H C}$ such that $J \phi_{n}=-\phi_{-n}$ for all positive $n$. Let $C$ be the set of all Hilbert-Schmidt operators on $\mathfrak{F C}$ such that $T^{*} J=-J T$. Then $C$ is the set of all $T \in K$ which are skew-symmetric with respect to the skew-symmetric form $\langle x, y\rangle=(x, J y)$. A simple system of roots is given by

$$
\left\{2 \lambda_{1}, \lambda_{i}-\lambda_{i+1}: i=1,2, \cdots\right\} \text {. }
$$

3.7. Theorem 3. Let $L$ be a separable simple $L^{*}$ algebra which has a Cartan decomposition relative to some Cartan subalgebra. Then (up to a multiple of the inner product on $L) L$ is isomorphic to one of the following algebras:

(1) A, the algebra of all Hilbert-Schmidt operators on a separable Hilbert space $\mathfrak{H C}$.

(2) B, the algebra of all Hilbert-Schmidt operators $T$ on $\mathcal{H C}$ such that $T^{*} J$ $=-J T$ for some fixed conjugation $J$ of $\mathcal{H C}$.

(3) $C$, the algebra of all Hilbert-Schmidt operators $T$ on $\mathcal{H C}$ such that $T^{*} J$ $=-J T$ for some fixed anti-conjugation $J$ of $\mathcal{H C}$.

REMARK. It still should be shown that the remaining three algebras $A$, 
$B, C$ are nonisomorphic. For two algebras $L, L^{\prime}$ of the type described in 3.7 and acting on the same space $\mathfrak{H}$ let $L$ be equivalent to $L^{\prime}$ if and only if there is a unitary $U$ on $\Re_{C}$ with $U L U^{-1}=L^{\prime}$. We will show that $L$ and $L^{\prime}$ are isomorphic only if they are equivalent. Since $A, B$, and $C$ are clearly not equivalent this will be sufficient.

An $x \in L$ will be called primitive if (i) $x=x^{*} \neq 0$, (ii) $D_{x}^{3}=D_{x}$, and (iii) $x$ cannot be written $x=y+z$ where $y$ and $z$ satisfy (i) and (ii). By using the fact (see the proof of Theorem 2) that every Cartan subalgebra of $L$ is a set of diagonal matrices relative to some basis of $\mathfrak{H C}$ it follows that each such subalgebra has a basis of primitive elements and the vectors $h_{\alpha}$ are obtained from these by linear operations in a unique way according to the type of the associated graph. Since any isomorphism of $L$ will preserve primitive elements the set $\left\{h_{\alpha}: \alpha \in R\right\}$, and hence the graph of $L$, is determined up to equivalence and the same will then hold for $L$.

\section{Some remarks on derivations.}

Definition. Let $L$ be a semi-simple $L^{*}$ algebra. A bounded operator $D$ on $L$ will be called a derivation of $L$ if and only if $D[x, y]=[D x, y]+[x, D y]$ for all $x, y$ in $L$.

If $\operatorname{dim} L$ is finite it is known that every derivation of $L$ is inner, i.e. equal to $D_{x}$ for some $x \in L$ [3, Exposé 7]. However, this is not true in general. To see this let $A$ be the $L^{*}$ algebra of all Hilbert-Schmidt operators on a separable infinite-dimensional Hilbert space. Then $A$ is an associative ideal in the algebra of all bounded operators (see [4, pp. 73-75]). For a bounded operator $B$ let $T_{B}$ be the operator on $A$ defined by $T_{B} X=B X-X B$. Then $T_{B}$ is a bounded derivation of $A$ and $T_{B}=0$ if and only if $B$ is a scalar multiple of the identity. Hence $T_{B}$ is inner only if it differs from a Hilbert-Schmidt operator by a multiple of the identity and this implies $A$ has outer derivations. Similar arguments can be used for $B$ and $C$.

The same example can be used to show that the image of $L$ under the adjoint representation need not be closed. By the closed graph theorem this is equivalent to proving that the norms on $L$ and its image are not equivalent. Letting $L=A$ as above and regarding $A$ as a matrix algebra with the usual unit matrices as a basis let $X_{k}=k^{-1 / 2} \sum_{1}^{k} E_{i i}$. Then $\left\|X_{k}\right\|=1$ while $\left\|D_{X_{k}}\right\|$ $=k^{-1 / 2}$. Thus $\left\|D_{X_{k}}\right\|$ tends to zero as $k$ becomes large.

\section{BIBLIOGRAPHY}

1. W. Ambrose, Structure theorems for a special class of Banach algebras, Trans. Amer. Math. Soc. vol. 57 (1945) pp. 364-386.

2. Garrett Birkhoff, Analytical groups, Trans. Amer. Math. Soc. vol. 43 (1938) pp. 61-101.

3. Séminaire Sophus Lie, Théorie des algèbres Lie, Ecole Normale Supérieure, Paris, 1955.

4. R. Schatten, $A$ theory of cross spaces, Princeton University Press, 1950.

Massachusetts Institute of Technology, Cambridge, Massachusetts 